\title{
Comparison of In Vitro Release Rates of Diclofenac Topical Formulations Using an In-Line Cell Automated Diffusion System
}

\author{
Yogesh Upadhyay ${ }^{1}$, Abhishek Kumar Singh ${ }^{1}$, Sanjeev Mishra ${ }^{1}$, Sanjay Jagannath Gurule ${ }^{1}$, Arshad H. \\ Khuroo $^{1}$, Neeta Tiwari², and Simrata Bedi ${ }^{2}$ \\ ${ }^{1}$ Department of Clinical Pharmacology and Pharmacokinetics, Sun Pharmaceutical Industries Ltd., Gurugram, Haryana, India. \\ ${ }^{2}$ Department of Product Development and Research, Sun Pharmaceutical Industries Ltd., Gurugram, Haryana, India.
}

e-mail: Yogesh.Upadhyay@sunpharma.com; yogeshdus@gmail.com

\section{ABSTRACT}

The in vitro release test (IVRT) is a powerful tool for prediction of the impact of formulation excipients on the drug release profile as well as lot-to-lot uniformity. We have determined and compared the release profile of diclofenac (a non-steroidal anti-inflammatory agent) from marketed topical formulations using an in-line cell automated diffusion system. We describe the advantages of the automated diffusion system along with factors influencing the in vitro release, such as type of membranes and receptor solution. A validated reverse phase-high performance liquid chromatography (RP-HPLC) method at a wavelength of $276 \mathrm{~nm}$ in the range of 0.200 to $100.000 \mu \mathrm{g} / \mathrm{mL}$ was used for effective analysis of in vitro release samples, with good sensitivity and precision. A linear relationship was found, with a coefficient of determination $\left(R^{2}\right) \geq 0.90$ for diclofenac semisolid matrices. Among all similar strength formulations, the maximum release rate was obtained from Volini gel and Voltaren Forte, having $1 \% \mathrm{w} / \mathrm{w}$ and $2 \% \mathrm{w} / \mathrm{w}$ diclofenac, respectively.

KEYWORDS: In vitro release test (IVRT); diclofenac; in-line cell; automated diffusion system, dissolution

\section{INTRODUCTION}

D iclofenac has antipyretic and analgesic action and falls under the pharmacological category of non-steroidal anti-inflammatory drugs (NSAIDs). The analgesic effect of diclofenac is due to inhibition of prostaglandins synthesis, whereas the anti-inflammatory effect of diclofenac is believed to be due to inhibition of leukocyte migration and the enzyme cylooxygenase (COX-1 and COX-2) (1). Its antipyretic effects may be due to action on the hypothalamus, resulting in peripheral dilation, increased cutaneous blood flow, and subsequent heat dissipation (1). Diclofenac is used to treat pain, dysmenorrhea, ocular inflammation, osteoarthritis, rheumatoid arthritis, ankylosing spondylitis, and actinic keratosis (1).

Nowadays, importance of drug administration via the skin for quick therapeutic effects has increased. The permeation of chemicals through the skin can be measured by in vivo and in vitro techniques; however, the experimental conditions are simple for in vitro techniques. In contrast to the traditional oral route, the use of transdermal drug delivery bypasses first-pass metabolism of the liver, the acidic environment of the gastrointestinal tract, and problems of absorption in the stomach, which often contains food, resulting in inconsistent and pulsed delivery of drugs into the intestine and variability in plasma concentration-time profile.

In vitro release testing (IVRT) is one of the standard methods and valuable quality control tools that can be used to evaluate performance characteristics and compare batch-to-batch variations of semisolid formulations such as creams, gels, and ointments. Important changes (physical, chemical, manufacturing site, process of the supplier, or source of an active ingredient or excipient) in the characteristics of drug product or thermodynamic properties of the drug may show a difference in drug release; hence, the estimation of release rate (RLR) can predict the impact of these changes on drug release behavior (2).

IVRT for semisolid dosage forms is based on an openchamber diffusion cell system, usually fitted with a synthetic membrane. Here, the synthetic membrane acts as a non-rate-limiting barrier between the product and 
receptor solution. After the assembly setup and adequate equilibration of diffusion system, a pseudo-infinite dose of the desired product (test/reference) is applied to the upper side of the membrane in the open donor chamber of the diffusion cell. After dose application and over an appropriate time period, serially sampling of receptor solution is performed to generate an adequate release profile obtained from analyzing the drug content from applied topical formulations (3).

Advantages of IVRT include design prediction, quality assurance of desired formulation designing, optimization during early stages of development, and assessment of product sameness after post-approval changes. Furthermore, other advantages include setting regulatory specifications for the products for quality control purposes to ensure the impact of excipients on product performance, evaluation of potential dosage variations, and monitoring lot-to-lot consistency to ensure product sameness. Compared to other quality control tests like assay, homogeneity, rheological properties, specific gravity, and particle size determination, IVRT provides more information regarding the release properties for topical formulations; however, the test is not a measurement of or predictive for bioavailability (2).

IVRT regulatory guidance for industry from the United States (US) Food and Drug Administration (FDA) is available (4). This guidance is for semisolid dosage forms such as gels, ointments, creams, and lotions. The use of IVRT is also suggested in the United States Pharmacopeia (USP) General Chapter $\langle 1724>$, and the method is aligned with the US FDA guidance, as reported by Ueda et al $(4,5)$.

In this study, an in-line cell (ILC) automated diffusion system was used to estimate RLRs of said topical formulations. The main advantage of an ILC automated system over a manual system is the elimination of process variations related to the actual amount of sampling and its deviation in sampling time, with the ease of experiments. Further, due to the continuous flow principle of the ILC automated diffusion system, problems related to maintaining sink conditions and saturation throughout the experiment can be eliminated.

The main aim of this study was to provide a fast, automated, convenient, and reproducible technique for IVRT and comparison between various strengths of diclofenac topical formulations using an ILC automated diffusion system.

\section{In-Line Cell (ILC) Automated Diffusion System}

ILC automated diffusion systems are similar to a flow-type
Franz diffusion cell apparatus with a very small receptor volume. ILCS have the membrane of interest in the horizontal plane and open donor chambers. The cells consist of an inlet compartment, a donor compartment, and a receptor compartment.

This instrument is designed for measurement of flux over time (experiments are based on continuous flow principle). A peristaltic pump having multiple channels carries the receptor solution from a reservoir through a distribution manifold and sends it to the ILCs. ILC systems from PermeGear have a fraction collector and a heating circulator bath to warm the ILCs to a desired temperature (i.e., $32 \pm 1.0^{\circ} \mathrm{C}$ ). Scintillation vials can be used for collection of release samples at set time intervals. A schematic diagram of an ILC automated diffusion system is shown in Figure 1.



Figure 1. Schematic diagram of in-line cell automated diffusion system.

After filling the receptor chambers of the cells and purging the system of bubbles, predose samples are collected after desired equilibrations of employed synthetic membranes. The semisolid matrix is then placed in the donor chambers of the cells. Further, the fluid is collected in the scintillation vials of the fraction collector. The scintillation vials are taken for analysis manually after the sampling time has elapsed. Replenishing is not required in ILCs after collection of each time interval as the receptor solution flowing continuously through donor cells (6).

\section{Reaction Order Kinetics}

The release of active moiety from its pharmaceutical formulation can be demonstrated by three mathematical models, i.e. zero-order kinetics, first-order kinetics, 
and Higuchi model (7). A Higuchi model is generally used for the estimation of RLR of topical semisolid preparations, and it has the several assumptions: initial drug concentration in the matrix is much higher than the drug solubility; diffusion of the drug should be in only one dimension; sink conditions should be maintained during the release period; and there should be no matrix swelling (7). According to Higuchi, there should be a linear relationship between the amount of drug released/ diffused $(Q)$ and the square root of time, with a coefficient of determination $\left(R^{2}\right) \geq 0.90(8)$.

\section{Synthetic Membranes}

Permeation experiments are normally carried out with excised human or animal skin; however, synthetic membranes are employed for release testing. The synthetic membrane should be commercially available with minimum cost, should show minimum binding with drug and minimum resistance for diffusion, and should be inert and compatible with the respective receptor solution. As per USP, synthetic membranes are chosen to offer the least possible diffusional resistance and not to be rate controlling (9).

\section{Receptor Solution}

Selection of a receptor solution should be based on the solubility and stability of the active drug in the medium. Ideally, receptor media should be aqueous systems and for most studies, isotonic solutions, buffered to a $\mathrm{pH}$ of 7.4. However, for products formulated with waterinsoluble drugs, the selection of an appropriate receptor solution to maintain sink conditions is a challenge. It may be necessary to alter receptor fluid $\mathrm{pH}$, add surfactants and/or complex agents, or use organic media in which the drug is more soluble to efficiently dissolve the active pharmaceutical ingredient (API) during release studies $(2,9)$.

\section{Sample Application and Membrane Temperature}

For IVRT experiments, an infinite dose is preferred over a finite dose for the following main reasons. An infinite dose simplifies diffusion kinetics, reduces variability due to slight mass variations in finite dosing, and makes application of dose easier (weighing not necessary). The temperature of a membrane may be changed as per the specific site of action, e.g., vaginal creams may be tested at $37 \pm 1.0^{\circ} \mathrm{C}$ (9). Donor cells should be occluded during the experiment as air exposure can affect the release rate (2).

\section{Reverse Phase-HPLC}

A variety of general validation protocols have been recommended by organizations, such as the US FDA, USP, and the International Conference on Harmonization (ICH). RP-HPLC is a commonly used powerful and reliable analytical tool for in vitro analysis of complex formulations such as creams, ointments, and gels.

\section{MATERIALS AND METHODS}

\section{Materials}

Various diclofenac topical formulations were used for IVRT experiments and are listed in Table 1. Working standard diclofenac diethylamine was from Aarti Drugs Ltd., Mumbai, India. HPLC-grade acetonitrile and methanol were from Sigma Aldrich Chemical Private Ltd., Bengaluru, India. Formic acid and ammonium acetate were from Thermo Fisher Scientific, Mumbai, India. Phosphatebuffered saline was from Sisco Research Laboratories, Mumbai, India. Synthetic membranes (Ultipor $\mathrm{N}_{66}$, Nylon 6,6 [0.2 $\mu \mathrm{m} \times 25 \mathrm{~mm}$, Lot No. IN15000705; $0.2 \mu \mathrm{m} \times 25$ $\mathrm{mm}$, Lot No. IN12000094), Tuffryn HT-200, (0.2 $\mathrm{m} \times 25$ $\mathrm{mm}$, Lot No. T30120), Supor $200(0.2 \mu \mathrm{m} \times 25 \mathrm{~mm}$, Lot No. IN14000045]) were from Pall Life Sciences, Mumbai, India. In vitro release tests were performed using ILC automated diffusion system from PermeGear, Pennsylvania, USA, equipped with peristaltic pump from Ismatech, Mumbai, India. For temperature monitoring of ongoing experiment, infrared thermometer from Metravi, MT-4, West Bengal, India was used. The analysis was performed in Analyst 1.4.2 software installed in HPLC system equipped with UV detector from Shimadzu, Mumbai, India.

\section{RP-HPLC}

RP-HPLC method validation was performed in the range of 0.201 to $100.103 \mu \mathrm{g} / \mathrm{mL}$ at $276 \mathrm{~nm}$ using Gemini NX C18 $(100 \times 4.6 \mathrm{~mm}, 5 \mu \mathrm{m})$ column (Phenomenex, Hyderabad, India) with Buffer 1 ( $20 \mathrm{mM}$ ammonium acetate $+8 \mathrm{~mL}$ formic acid per liter) in combination with acetonitrile, $45: 55 \mathrm{v} / \mathrm{v}$ ) as mobile phase with $0.700 \mathrm{~mL} / \mathrm{min}$ flow rate, $10-\mu \mathrm{L}$ injection volume, and column oven temperature set as $35^{\circ} \mathrm{C}$.

\section{ILC Automated Diffusion System Assembly Setup Procedure}

The IVRT experiment was performed using an ILC automated diffusion cell system as follows. The heating circulator bath was turned on, and the temperature of the water tank was set at $45^{\circ} \mathrm{C}$ to maintain membrane surface temperature at about $32 \pm 1{ }^{\circ} \mathrm{C}$. The reservoir bottle and distribution manifold were connected, and the reservoir bottle was filled with receptor solution $(10 \mathrm{mM}$ phosphate buffer saline solution). The peristaltic pump's tubing was connected to the distribution manifold and to ILCs. The flow rate of $0.100 \mathrm{~mL} / \mathrm{min}$ and dispensing time 
Table 1. Details of Formulations Used for IVRT Experiments

\begin{tabular}{|c|c|c|c|c|}
\hline Formulation Name & Active Substance & Strength $(\% \mathrm{w} / \mathrm{w})$ & Marketed By & Batch or Lot No. \\
\hline Volini Gel & Diclofenac diethylamine & 1.16 & $\begin{array}{l}\text { Sun Pharmaceutical, } \\
\text { Mumbai, Maharashtra, } \\
\text { India }\end{array}$ & 2716272 \\
\hline Vicks Multipain Relief Gel & Diclofenac diethylamine & 1.16 & $\begin{array}{c}\text { Procter \& Gamble, Mumbai, } \\
\text { Maharashtra, India }\end{array}$ & 4192050038 \\
\hline Voveran ThermaGel & Diclofenac sodium & 1 & $\begin{array}{l}\text { Novartis, Hyderabad, } \\
\text { Telangana, India }\end{array}$ & $145009 \mathrm{~KB}$ \\
\hline Iodex Ultra Gel & Diclofenac diethylamine & 1.16 & $\begin{array}{l}\text { GlaxoSmithKline, Mumbai, } \\
\text { Maharashtra, India }\end{array}$ & B4006 \\
\hline Voltaren Forte & Diclofenac diethylamine & 2.32 & $\begin{array}{c}\text { GlaxoSmithKline, Mumbai, } \\
\text { Maharashtra, India }\end{array}$ & P04971A \\
\hline Dicloran Gel & Diclofenac sodium & 2 & $\begin{array}{c}\text { JB Chemicals and } \\
\text { Pharmaceutical Ltd., } \\
\text { Mumbai, Maharashtra, } \\
\text { India }\end{array}$ & XD1503 \\
\hline Nacgel & Diclofenac diethylamine & 2.32 & $\begin{array}{l}\text { Systopic Laboratories } \\
\text { Private Ltd., New Delhi, } \\
\text { India }\end{array}$ & M01 10/13 \\
\hline
\end{tabular}

Note: $1.16 \%$ and $2.32 \%$ of diclofenac diethylamine is equivalent to $1 \%$ and $2 \%$ of diclofenac sodium, respectively.

was programmed on the peristaltic pump. The flow rate was selected after optimizing the method. Six ILCs were placed in the cell warmer, and tubing from peristaltic pump was connected to each inlet compartment. Selected synthetic membrane (Supor 200 [13 mm diameter, $0.2 \mu \mathrm{m}]$ ] was placed into the cell body, and the donor chamber was placed over it. The receptor solution was purged, ensuring no air bubbles remained inside the tubing and below the membrane. The fraction collector was programmed, and the samples were collected in glass scintillation vials. The temperature on the membrane surface was measured prior to sample application via an infrared thermometer.

\section{Selection of Receptor Solution}

RLRs with $R^{2}$ of diclofenac formulations were evaluated by using various receptor solutions compositions and components. The solubility of diclofenac was found to be excellent in phosphate buffer saline. Solubility of the analyte in the receptor solution was tested. The solubility of diclofenac in receptor solution was found to be 835.591 $\mu \mathrm{g} / \mathrm{mL}$ (calculated theoretical yield was $858.801 \mu \mathrm{g} / \mathrm{mL}$ ), and $97.30 \%$ diclofenac was calculated as recovery.

\section{Selection of Synthetic Membrane Using Membrane Binding Test Method}

This test was performed to determine the suitability of synthetic membrane in providing inertness for the diffusion of drug across it from a semisolid dosage form. Supor, Ultipor, and Tuffryn synthetic membranes were used to evaluate membrane binding. Among the screened membranes, the Ultipor N66 (Nylon 6,6) and Tuffryn HT-200 membranes showed significant binding (20.19\% and $15.00 \%$, respectively) of diclofenac on the membrane, whereas Supor 200 provided the least binding (5.64\%). The higher recovery (94.36\%) indicated that the Supor 200 membrane provided more inertness in the diffusion of diclofenac from the formulation across it and is therefore more suitable for IVRT experiments.

\section{Formulation Application and Sample Collection}

During membrane equilibration and prior to formulation application, predose samples were collected in the first set point to ensure no interference was coming from assembly setup or membrane or receptor solution at retention time (RT) of API. As soon as the cell warmer moved to the second set point, at first sampling time point of $6 \mathrm{~min}$, approximately $500 \mu \mathrm{L}$ (infinite dose) of the desired topical formulation was applied onto the membrane surface of each ILC using a dosing syringe and evenly spread as required $(n=6)$. The donor chambers of each ILC were occluded with a parafilm. The spring clamp was placed over the donor chamber and screwed with nuts. IVRT samples were collected in scintillation vials over the set sampling time points (i.e., predose, $6,12,24,36,48$, and $60 \mathrm{~min})$. After completion of IVRT experiment, all collected time point samples were diluted to four folds using the receptor solution to achieve 
quantifiable concentrations within analytical range. The release concentrations at each sampling time point were obtained through HPLC analysis. The amount released at each sampling time point $\left(\mu \mathrm{g} / \mathrm{cm}^{2}\right)$ was calculated as follows: concentration obtained at each sampling time point $(\mu \mathrm{g}) \times$ time interval $(\mathrm{min}) \times$ flow rate $(\mathrm{mL} / \mathrm{min})$ divided by the effective surface area of membrane $(0.785$ $\mathrm{cm}^{2}$ ). Amount of drug released was summed to get the cumulative amount released.

The in vitro RLR was calculated by plotting the cumulative amount of drug release per unit area $\left(\mu \mathrm{g} / \mathrm{cm}^{2}\right)$ against the square root of time $(\mathrm{Vt})$ which yields a straight line, the slope of which gives RLR.

\section{Selection of Sampling Time Points}

After mounting of membranes on ILCs, membranes mounted in each donor chamber were equilibrated for $30 \mathrm{~min}$. To plot actual linearity, a minimum of six sampling time points is required (7). Due to the high solubility of diclofenac in alkaline medium (i.e., phosphate buffer saline), 1 hour experimental duration for calculating release rates was found to be suitable. The discriminative results were found for the $1 \%$ and $2 \%$ strength diclofenac formulations by determining release rates at $6,12,24,36$, 48 , and 60 mins.

\section{RESULTS AND DISSCUSION}

\section{Linearity and Range}

The linearity of the method was determined by analysis of standard plots associated with an eight-point standard calibration curve. The calibration curve was found to be linear $(y=32100 x+-286)$ from $0.201 \mu \mathrm{g} / \mathrm{mL}$ [limit of quantitation (LOQ)] to $100.103 \mu \mathrm{g} / \mathrm{mL}$ [upper limit of quantitation (ULOQ)] with correlation coefficient $(r)$ as 0.9998. Back-calculations were made from that curve to determine concentrations of diclofenac in unknown samples.

\section{Selectivity Using Synthetic Membrane}

The synthetic membrane (Supor 200) was dipped in the receptor solution for $60 \mathrm{~min}$ (covering the entire duration of the experiment). The peak area responses obtained at the RT of analyte in each synthetic membrane release blank sample were evaluated. No significant interfering peaks from synthetic membrane released blank samples were observed at the RT of diclofenac. This indicates that the analytical method is selective for the analysis of diclofenac.

\section{Precision and Accuracy}

Precision and accuracy expressed as a percentage of deviation from the respective nominal value and the precision of the assay were measured by the percentage coefficient of variation (CV) at each concentration. Intraday precision and accuracy were assessed by analyzing 12 replicates of the quality control $(\mathrm{QC})$ samples on the same day at four levels of QC (i.e., $0.206,0.584,40.0$, and $80.0 \mu \mathrm{g} / \mathrm{mL}$ for LOQ [LOQQC], low, medium, and high QC) prepared in receptor solution. The inter-day precision and accuracy were assessed by analyzing 12 replicates of the quality control samples at each above-mentioned QC level prepared in receptor solution through two precision and accuracy batches run on two consecutive validation days.

The deviation at each concentration level from the nominal concentration was found to be within 97.3299.26\% for intra-day and $96.79-98.33 \%$ for inter-day accuracy. Similarly, the mean precision was found to be within $0.82-1.07$ for intra-day and $0.78-1.97$ for inter-day.

\section{IVRT}

Results of IVRT are summarized in Table 2. A robust, fast, automated, reproducible, and precise in vitro release test was developed and applied to determine in vitro RLR of diclofenac marketed formulations label claimed as $1 \%$ $\mathrm{w} / \mathrm{w}$ and $2 \% \mathrm{w} / \mathrm{w}$ of diclofenac using an ILC automated diffusion system. IVRT was carried out for 60 min with six automated sampling time points. For formulations with diclofenac $1 \% \mathrm{w} / \mathrm{w}$, the order of drug release (average $\mathrm{RLR}, n=6$ ) from different gel formulations was Volini Gel $>$ Vicks Multipain Relief Gel > lodex Ultra Gel > Voveran ThermaGel. $R^{2}$ for all formulations were found to be greater than 0.90 , showing linear and consistent release from each ILC over a 1-hour sampling period. Percent CV was calculated between ILCS $(n=6)$ and found to be less than 15\% (Figs. 2 and 3). For diclofenac 2\% w/w formulations, order of drug release (average of RLR, $n=6$ ) was Voltaren Forte $>$ Dicloran Gel $>$ Nacgel, with $R^{2}>0.90$ and percent CV less than 15\% (Figs. 4 and 5).

\section{CONCLUSION}

For the pharmacological action of a topical dermal drug product to occur, the drug must first be released from the vehicle to be available to penetrate the skin layers and reach the site of action. Drug release is mainly dependent on the characteristics of the formulation, and IVRT is the major tool for assessing drug release rates and predicting the impact of the excipients and manufacturing processes on drug release. Use of an ILC automatic diffusion systems provides automated sampling, time-saving, and accurate discriminative results between all formulations. 
Table 2. Comparative Results of IVRT Experiments from all Diclofenac Topical Formulations

\begin{tabular}{|c|c|c|c|c|c|c|c|c|c|c|}
\hline \multirow{2}{*}{ Formulation Name } & \multirow{2}{*}{ Parameters } & \multicolumn{6}{|c|}{ In-Line Cell No. } & \multirow{2}{*}{ Mean } & \multirow{2}{*}{ SD } & \multirow{2}{*}{ CV } \\
\hline & & 1 & 2 & 3 & 4 & 5 & 6 & & & \\
\hline \multicolumn{11}{|c|}{$1 \% \mathrm{w} / \mathrm{w}$ Strength Formulations } \\
\hline \multirow{2}{*}{ Volini Gel } & RLR & 251.3 & 255.7 & 241.1 & 296.8 & 239 & 240.7 & \multirow{2}{*}{254.10} & \multirow{2}{*}{21.956} & \multirow{2}{*}{$8.65 \%$} \\
\hline & $R^{2}$ & 0.9903 & 0.9832 & 0.9818 & 0.9905 & 0.9835 & 0.9801 & & & \\
\hline \multirow{2}{*}{$\begin{array}{l}\text { Vicks Multipain } \\
\quad \text { Relief Gel }\end{array}$} & RLR & 159.0 & 160.4 & 194.8 & 145.3 & 127.1 & 156.6 & \multirow{2}{*}{157.20} & \multirow{2}{*}{22.250} & \multirow{2}{*}{$14.15 \%$} \\
\hline & $R^{2}$ & 0.9794 & 0.9574 & 0.9644 & 0.9743 & 0.9788 & 0.9657 & & & \\
\hline \multirow{2}{*}{$\begin{array}{l}\text { Voveran } \\
\text { ThermaGel }\end{array}$} & RLR & 120.9 & 130.6 & 144.4 & 144.8 & 103.9 & 128.9 & \multirow{2}{*}{128.92} & \multirow{2}{*}{15.394} & \multirow{2}{*}{$11.94 \%$} \\
\hline & $R^{2}$ & 0.9379 & 0.9318 & 0.9261 & 0.9156 & 0.9063 & 0.9206 & & & \\
\hline \multirow{2}{*}{ Iodex Ultra Gel } & RLR & 134.7 & 137.7 & 132.8 & 132.68 & 129.8 & 114.8 & \multirow{2}{*}{130.41} & \multirow{2}{*}{8.080} & \multirow{2}{*}{$6.20 \%$} \\
\hline & $R^{2}$ & 0.9717 & 0.9623 & 0.9638 & 0.9646 & 0.9514 & 0.9817 & & & \\
\hline \multicolumn{11}{|c|}{$2 \% \mathrm{w} / \mathrm{w}$ Strength Formulations } \\
\hline \multirow{2}{*}{ Voltaren Forte } & RLR & 367.6 & 412.1 & 283.0 & 367.8 & 354.5 & 337.7 & \multirow{2}{*}{353.78} & \multirow{2}{*}{42.562} & \multirow{2}{*}{$12.03 \%$} \\
\hline & $R^{2}$ & 0.9406 & 0.9800 & 0.9865 & 0.9765 & 0.9716 & 0.9935 & & & \\
\hline \multirow{2}{*}{ Dicloran Gel } & RLR & 310.8 & 318.5 & 326.5 & 348.4 & 304.1 & 349.2 & \multirow{2}{*}{326.25} & \multirow{2}{*}{19.009} & \multirow{2}{*}{$5.83 \%$} \\
\hline & $R^{2}$ & 0.9856 & 0.9859 & 0.9863 & 0.9875 & 0.9881 & 0.9883 & & & \\
\hline \multirow{2}{*}{ Nacgel } & RLR & 294.2 & 314.7 & 298.1 & 277.7 & 281.8 & 256.5 & \multirow{2}{*}{287.17} & \multirow{2}{*}{19.930} & \\
\hline & $R^{2}$ & 0.9179 & 0.9432 & 0.9531 & 0.9226 & 0.9441 & 0.9211 & & & $6.94 \%$ \\
\hline
\end{tabular}

$R L R$, release rate $\left(\mu \mathrm{g} / \mathrm{cm}^{2} / h^{1 / 2}\right) R^{2}$, coefficient of determination; $C V$, coefficient of variation

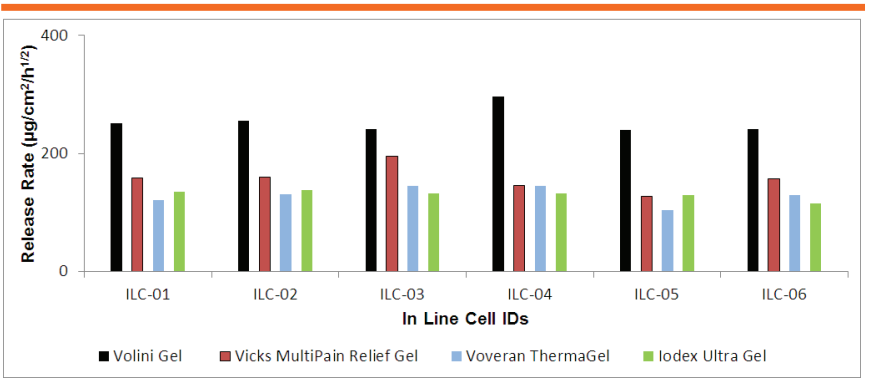

Figure 2. Release rates (RLRs) for $1 \% \mathrm{w} / \mathrm{w}$ diclofenac formulations. ILC, in-line cells.

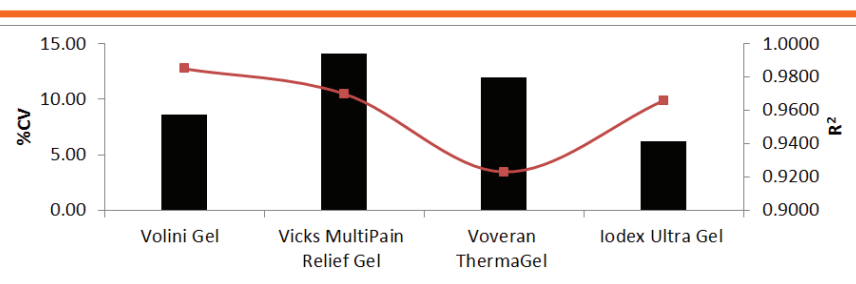

Formulations Name

$\% \mathrm{CV} \rightarrow \mathrm{R}^{2}$

Figure 3. Coefficient of variation (CV) and determination $\left(R^{2}\right)$ obtained from $1 \% \mathrm{w} / \mathrm{w}$ diclofenac formulations $(n=6)$.

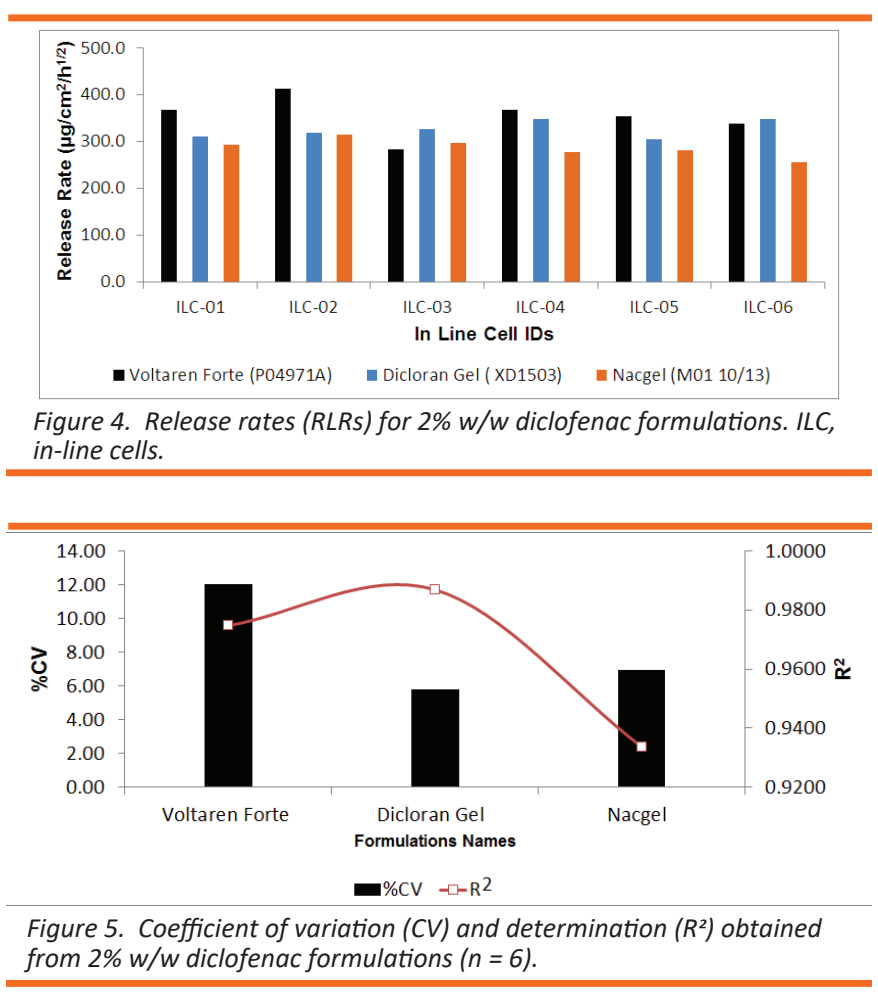

|Dissolution 
The objective of this study was to determine a fast and accurate technique for IVRT of different diclofenac topical formulations having $1 \% \mathrm{w} / \mathrm{w}$ and $2 \% \mathrm{w} / \mathrm{w}$ strengths using an ILC automated diffusion system. The developed methodology can discriminate release rates between topical formulations using an ILC automated system. As an added advantage, this validated HPLC and automated IVRT method can be employed for qualitative and quantitative release rate analysis of other topical diclofenac formulations as well.

Among all similar strength formulations, the maximum release rate was obtained from Volini gel and Voltaren Forte, having $1 \% \mathrm{w} / \mathrm{w}$ and $2 \% \mathrm{w} / \mathrm{w}$ diclofenac, respectively. The minimum coefficient of variation $<$ $15 \%)$ shows less intra-cell variability between ILCs, leading to accurate release rate analysis for each formulation. Results also indicate that the thermodynamic property of the drug would not fluctuate greatly within the applied temperature range (i.e., $32 \pm 1{ }^{\circ} \mathrm{C}$ ).

\section{CONFLICTS OF INTEREST}

The authors disclosed no conflicts of interest related to this article.

\section{REFERENCES}

1. Ulubay, M.; Yurt, K. K.; Kaplan, A. A.; Atilla, M. K. The use of diclofenac sodium in urological practice: $A$ structural and neurochemical based review. J. Chem. Neuroanat. 2018, 87, 32-36. DOI: 10.1016/j.jchemneu.2017.02.005.

2. Klein, R. R.; Tao, J. Q.; Wilder, S.; Burchett, K.; Bui, Q.; Thakker,
K. D. Development of an in vitro release test (IVRT) for a vaginal microbicide gel. Dissolution Technol. 2010, 17, 6-10. DOI: 10.14227/DT170410P6.

3. Kanfer, I.; Rath, S.; Purazi, P.; Mudyahoto, N. A. In vitro release testing of semi-solid dosage forms. Dissolution Technol. 2017, 24, 52-60. DOI: 10.14227/DT240317P52.

4. Nonsterile Semisolid Dosage Forms, Scale-Up and Postapproval Changes: Chemistry, Manufacturing, and Controls; In Vitro Release Testing and In Vivo Bioequivalence Documentation; Guidance for Industry; U.S. Department of Health and Human Services, Food and Drug Administration, Center for Drug Evaluation and Research (CDER), U.S. Government Printing Office: Washington, DC, 1997. DOI: 10.1201/9780824741969. axe.

5. Ueda, C. T.; Shah, V. P.; Derdzinski, K.; Ewing, G.; Flynn, G.; Maibach, H.; Marques, M.; Rytting, H.; Shaw, S.; Thakker, K.; Yacobi, A. Topical and transdermal drug products. Dissolution Technol. 2010, 17, 12-25. DOI: 10.14227/DT170410P12.

6. PermeGear Automated Systems website. http://permegear. com/automated-systems (accessed December 24, 2018).

7. Dash, S.; Murthy, P. N.; Nath, L.; Chowdhury, P. Kinetic modeling on drug release from controlled drug delivery systems. Acta Pol. Pharm. 2010, 67, 217-223.

8. Higuchi, T. Rate of release of medicaments from ointment bases containing drugs in suspension. J. Pharm. Sci. 1961, 50, 874-875. DOI: 10.1002/jps.2600501018.

9. Olejnik, A.; Goscianska, J.; Nowak, I. Active compounds release from semisolid dosage forms. J. Food Drug. Anal. 2012, 101, 4032-4045. DOI: 10.1002/jps.23289. 